\title{
O que se tem pesquisado sobre Sustentabilidade Empresarial e sua Evidenciação?
}

\begin{abstract}
Resumo
Objetivo: $\mathrm{O}$ objetivo deste artigo é apresentar, por meio de uma revisão da literatura, a evolução do conceito de desenvolvimento sustentável e sustentabilidade no decorrer dos anos e as tendências nas pesquisas com foco na intersecção entre Contabilidade e Sustentabilidade.

Método: $\mathrm{O}$ estudo foi desenvolvido por meio da revisão das literaturas nacional e internacional sobre sustentabilidade e seus relacionamentos com a Contabilidade. A seleção da literatura pesquisada priorizou publicações mais recentes; um misto entre pesquisas empíricas e revisões de literaturas; journals que apresentavam algum fator de impacto e periódicos nacionais qualificados no Qualis/Capes.

Resultados: Pesquisas vêm avançando especialmente em dois focos: a) evidenciação - são muitas pesquisas que buscam verificar a quantidade e a qualidade de disclosure sobre a sustentabilidade, inclusive com sugestões de modelos como o Relato Integrado; e b) busca de relação entre práticas e indicadores de sustentabilidade com desempenho econômicofinanceiro das empresas. Os resultados têm se mostrado muitas vezes controversos, o que abre espaços para novas pesquisas mais robustas, com teorias de base mais consistentes.

Contribuições: As pesquisas sobre o tema Sustentabilidade são recentes e foram intensificadas nos últimos 20 anos, tornandose importante que haja um estudo que busque refletir como vêm evoluindo os achados dessas pesquisas, especialmente em questões que ainda não se tem resultados consolidados. Assim, a contribuição deste artigo vem com a forma de se estruturar a construção do conhecimento científico sobre sustentabilidade e, com isso destacar lacunas que ainda necessitam ser estudadas em futuras pesquisas.
\end{abstract}

Palavras-chave: Sustentabilidade; Relato Integrado; Indicadores de Sustentabilidade.

\section{Valcemiro Nossa}

Doutor em Controladoria e Contabilidade pela Universidade de São Paulo (USP) e Professor na Fucape Business School. Contato: Av. Fernando Ferrari, 1358. Boa Vista. Vitória (ES). CEP: 29075-505. E-mail: valcemiro@fucape.br

Victor Rangel dos Santos Rodrigues

Graduando em Economia na Fucape Business School. Contato: Av. Fernando Ferrari, 1358. Boa Vista. Vitória (ES). CEP: 29075-505.

E-mail: victorrangelrodrigues@gmail.com

\section{Silvania Neris Nossa}

Doutora em Ciências Contábeis e Administração pela Fucape Business School e Professora Associada na Fucape Business School. Contato: Av. Fernando Ferrari, 1358. Boa Vista. Vitória (ES). CEP: 29075-505.

E-mail: silvanianossa@fucape.br 


\section{Introdução}

A ideia de desenvolvimento sustentável está no cerne das aspirações da humanidade neste século. Tal afirmação é confirmada pelo crescimento exponencial de estudos relacionados ao tema e, por movimentos como os Objetivos de Desenvolvimento Sustentável e os Objetivos do Milênio, ambos da ONU, terem rápida aceitação devido à urgência por práticas que não agridam ao meio ambiente e tragam sustentabilidade também nos âmbitos social e econômico (Allen, Metternicht \& Wiedmann, 2016; Campbell, 2017; Griggs, Stafford-Smith, Gaffeny, Rockström, Ohman, Shyamsundar \& Noble, 2013; Sachs, 2012).

O surgimento do conceito de Sustentabilidade está interligado ao de Desenvolvimento Sustentável, que emergiu de um contexto de preocupações com os impactos da produção e ações humanas no meio ambiente, a escassez de recursos naturais e o futuro no mundo, expressado em documentos, como o conhecido relatório emitido pela Comissão Mundial de Meio Ambiente e Desenvolvimento (1987). Nesse relatório, desenvolvimento sustentável é definido como "o desenvolvimento que satifaz às necessidades do presente sem comprometer a habilidade das futuras gerações de suprir suas próprias necessidades" (Bruntland, 1987, p. 8). Outros documentos, como o World Charter for Nature (1982) e a Agenda 21 da Earth Summit (1992), também expressam o conceito de desenvolvimento sustentável (Assembly, 1982; Brundtland, 1987; Hák, Janoušková, \& Moldan, 2016; Kopnina, 2015; Redclift, 2005; Summit, 1992; Wood, 1985).

Vários questionamentos derivaram da definição proposta. Por exemplo, para impedir que a satisafação da necessidade presente não comprometa as gerações futuras, é necessário conhecer as necessidades e habilidades das novas gerações. Porém, se a humanidade enfrenta diversas dificuldades em desenvolver um consenso global acerca das necessidades da geração presente, qual a possibilidade de desenvolver este mesmo consenso para as gerações futuras? Assim, a definição sugerida carece de aplicabilidade e não pode ser balizadora de decisões políticas e de negócios (Marshall \& Toffel, 2005).

A dificuldade de conceituar sustentabilidade advém da natureza dos problemas dessa área, pois se tratam de sistemas complexos, com variáveis de diversas áreas do conhecimento, correlacionadas de forma não linear. Por exemplo, o mainstream econômico diz que a chave para o desenvolvimento sustentável se encontra na abordagem da Economia do Bem-Estar Social. Outros cientistas com diferentes visões, como biólogos, economistas institucionais e ecológicos, tendem a discordar, trazendo conceitos e teorias advindos de suas respectivas áres de formação (Arrow, Dasgupta, Goulder, Daily, Ehrlich \& Summer, 2004; Ayres, 2008; Bithas, 2011; de Vries \& Petersen, 2009; Goodland \& Ledec, 1987; Saunders, 2014; Solow, 1993; Stavins, Wagner \& Wagner, 2003; Wagner, 2006).

$\mathrm{Na}$ literatura, abordagens divergem em relação ao método, porém compartilham, mesmo que basilarmente, da mesma preocupação de que, se não forem protegidas, as futuras gerações viverão em um ambiente mais hostil do que foi vivido no passado ou no presente. Assim, uma nova área de pesquisa surge, a Ciência da Sustentabilidade, com a característica de abordar os problemas de sistemas complexos de fatores correlacionados ligados à sustentabilidade e desenvolvimento sustentável de uma forma transdisciplinar (Brandt, Ernst, Gralla, Luederitz, Lang Newig \& Von Wehrden, 2013; Campbell, 2017; Caniglia, Schäpke, Lang, Abson, Luederitz \& Von Wehrden, 2017; Leal Filho, 2000; Gerlagh, 2017; Hellwig, 2005; Jerneck, Olsson, Ness, Anderberg, Baier, Clark \& Person, 2011; Kajikawa, 2008; Komiyama \& Takeuchi, 2006; Mikhailova, 2004; Miller, Armim, Sarewitz, Robinson, Olsson \& Loorbach, 2014; Pappas, Pappas, \& Sweeney, 2015; Phillis \& Andriantiatsaholiniaina, 2001; Sala, Farioli, \& Zamagni, 2013; Vallance, Perkins, \& Dixon, 2011).

Assim, o objetivo deste estudo é apresentar a evolução do conceito de desenvolvimento sustentável e sustentabilidade no decorrer dos anos e as tendências nas pesquisas com foco na intersecção entre Contabilidade e Sustentabilidade.

O estudo foi desenvolvido por meio da revisão da literatura nacional e internacional sobre Sustentabilidade e seus relacionamentos com a contabilidade. A seleção da literatura pesquisada priorizou publicações mais recentes; um misto entre pesquisas empíricas e revisões de literaturas; journals que apresentavam algum fator de impacto e periódicos nacionais qualificados no Qualis/Capes. 
Considerando que as pesquisas sobre o tema Sustentabilidade são recentes e foram intensificadas nos últimos 20 anos, torna-se importante que haja um estudo que busque refletir como vem evoluindo os achados dessas pesquisas, especialmente em questões que ainda não se têm resultados consolidados. Assim, a contribuição deste artigo vem como forma de se estruturar a construção do conhecimento científico sobre Sustentabilidade e, com isso, destacar lacunas que ainda necessitam ser estudadas em futuras pesquisas.

\section{Sustentabilidade e Desenvolvimento Sustentável}

A abordagem deste tópico incia-se com um breve descritivo do histórico dos conceitos de Sustentabilidade e Desenvolvimento Sustentável, destacando-se no tempo os principais eventos que levaram à evolução do conceito de Desenvolvimento Sustentável. Em seguida, são discutidas as diferentes abordagens do conceito e finaliza com os Obejtivos do Desenvolvimento Sustentável (ODS) propostos pela Organizações das Nações Unidas (ONU).

\subsection{Breve Histórico do Conceito}

As raízes do conceito da Sustentabilidade podem ser mapeadas até os tempos da Grécia Antiga, porém ganha destaque na história da humanidade com novas questões trazidas pelo desenvolvimento tecnológico, o massivo aumento do consumo de energia e o crescimento exponencial da população a partir da Primeira Revolução Industrial (Du Pisani, 2006; Wrigley, 2013).

O termo "Sustentabilidade" foi cunhado por Carlowitz e Rohr (1732), em Sylvicultura Oeconomica. Ao entender que a madeira era o principal insumo do século XVIII, usado tanto para combustível como para construção civil, naval, etc., Carlowitz propõe o uso sustentável das florestas, ou seja, dever-se-ia assegurar a existência de árvores jovens o suficiente para substituir a lenha que fora utilizada nos processos produtivos da economia (Carlowitz \& Rohr, 1732; Du Pisani, 2006; Grober, 2007; Klöpffer, 2003).

Outro autor destacado na literatura sobre desenvolvimento sustentável foi Thomas R. Malthus, que desenvolveu um modelo analítico do crecimento populacional e da consequência do ritmo deste crescimento na escassez de recursos e alimentos (Malthus, 1798).

Em Principles of political economy (1848), Jonh Stuart Mill dedica um curto capítulo ao conceito de "estado estacionário", no qual o autor argumenta que o desenvolvimento cultural e moral era uma condição separada do desenvolvimento e do crescimento econômico, confrontando a ideia vigente de que a sociedade humana poderia avançar apenas se a economia se expandisse. Portanto, o autor diverge da ideia de crescimento contínuo, argumentando que haveria uma condição estacionária de geração de riqueza, consumo, capital e massa populacional, porém não de desenvolvimento humano (Mill, 1848 apud Caradonna, 2014; Du Pisani, 2006; Wagner, 2006).

Em Man and Nature (1864), Marsh (1965) descreve como diferentes aspectos do meio ambiente foram afetados pela intervenção humana e como essas perturbações podem acabar transformando o planeta em um lugar inabitável, podendo inclusive levar à extinção da raça humana. A linha argumentativa de Marsh se propunha a mostrar que a humanidade não teria continuidade sem cuidar do meio ambiente (Marsh, 1965; Pádua, 2010).

Devido ao crescimento na exploração Mineral, no século XIX, foi crescente também a preocupação acerca da questão energética. Em The Coal Question (1866), Stanley Jevons argumentava que o uso descomedido dos recursos poderia consumir as reservas de carvão inglesas em cerca de um século (Jevons, 1866 apud Caradonna, 2014; Clark \& Foster, 2001). O século XX trouxe um misto de pessimismo e otimismo em relação ao desenvolvimento da humanidade e sustentabilidade. Os bons presságios trazidos pelo desenvolvimento científico-tecnológico foram trazidos pelas crises econômicas e as guerras mundiais da primeira metade do século, porém, na segunda metade, o debate acerca do desenvolvimento sustentável ganha força e importância global (Caradonna, 2014). 
Houve, por exemplo, uma divergência dentro da doutrina econômica acerca do papel e de como deveria ser a abordagem da ciência econômica para o problema da Sustentabilidade. Surgiram então duas correntes principais: a Economia Ambiental e a Economia Ecológica. A primeira aplica o arcabouço da teoria das Externalidades para analisar problemas de escassez e alocação dos recursos naturais, tendo, portanto, um viés mais unidisciplinar e heterodoxo; enquanto a segunda corrente tem uma abordagem transdisciplinar entre a economia, ecologia, física, etc., considerando o sistema econômico como parte integrante do sistema ecológico (Costanza, 1989; Costanza, Cumberland, Daly, Goodland, Norgaard, Kubiszewski \& Franco, 2014; Cropper \& Oates, 1992; Du Pisani, 2006; Field \& Field, 1997; Goodland, 1995; Illge \& Schwarze, 2009; Romeiro, 2010).

Nessa época também, diversas comissões, conferências e acordos ocorreram como parte de um esforço em busca de Desenvolvimento Sustentável. O Quadro 1 sumariza alguns dos principais eventos ocorridos:

\section{Quadro 1}

\section{Conferências, Comissões e Acordos Globais em prol do Desenvolvimento Sustentável}

\begin{tabular}{|c|c|}
\hline Evento & Descrição \\
\hline $\begin{array}{l}\text { 1972, Estocolmo, } \\
\text { Conferencia ONU para o } \\
\text { Meio Ambiente Humano e a } \\
\text { "Declaração de Estocolmo" }\end{array}$ & $\begin{array}{l}\text { É considerado um marco na história da cooperação internacional em questões } \\
\text { ambientais. O Programa das Nações Unidas para o Meio Ambiente (PNUMA) foi criado. } \\
\text { Também, foi publicado um decreto de salvaguarda do meio ambiente e das questões } \\
\text { sociais. Além disso, a ONU começa a discutir a relação entre a poluição do ar advinda da } \\
\text { ação humana e as consequentes mudanças climáticas globais. }\end{array}$ \\
\hline $\begin{array}{l}\text { 1980, PNUMA- Estratégia de } \\
\text { Conservação Mundial }\end{array}$ & $\begin{array}{l}\text { Documento criado pela União Internacional para a Conservação da Natureza, com } \\
\text { participação de cientistas e pesquisadores de diversas nacionalidades. Aparentemente, é } \\
\text { o primeiro documento internacional a usar o termo "desenvolvimento sustentável". }\end{array}$ \\
\hline $\begin{array}{l}\text { 1982, Assembleia Geral da } \\
\text { ONU, "Carta Mundial da } \\
\text { Natureza" }\end{array}$ & $\begin{array}{l}\text { Argumenta que a humanidade é parte da natureza e a que vida é dependente do } \\
\text { funcionamento ininterrupto dos sistemas naturais. Aponta que a humanidade } \\
\text { pode alterar a natureza e até extrair os recursos naturais por sua ação ou por suas } \\
\text { consequências, mas que também deve manter a estabilidade e a qualidade da natureza } \\
\text { e ainda conservar os recursos naturais. }\end{array}$ \\
\hline $\begin{array}{l}\text { 1983-1987, Comissão } \\
\text { Mundial do Meio Ambiente } \\
\text { e Desenvolvimento e } \\
\text { "Nosso Futuro Comum" }\end{array}$ & $\begin{array}{l}\text { Também conhecida como a Comissão Brundtland, a proposta era criar uma estrutura } \\
\text { conceitual para o desenvolvimento sustentável mundial. O documento produzido } \\
\text { foi chamado de "Nosso Futuro Comum", no qual defende que as gerações atuais } \\
\text { devem utilizar-se da natureza sem comprometer a habilidade das futuras gerações no } \\
\text { atendimento de suas próprias necessidades. }\end{array}$ \\
\hline $\begin{array}{l}\text { 1992, Rio de Janeiro, } \\
\text { Conferência ONU para } \\
\text { o Meio Ambiente e } \\
\text { Desenvolvimento }\end{array}$ & $\begin{array}{l}\text { A Eco-92, ou Cúpula da Terra foi uma grande conferência, com a participação de diversos } \\
\text { lideres globais e ONG's. A “Declaração do Rio" contém } 27 \text { princípios que deveriam } \\
\text { orientar a política em relação ao meio ambiente e ao desenvolvimento. A "Agenda 21" } \\
\text { oferece um quadro detalhado para a implementação do desenvolvimento sustentável. }\end{array}$ \\
\hline 1997, Protocolo de Quioto & $\begin{array}{l}\text { Um tratado ambiental adotado em } 1997 \text { e que virou lei em 2005, que estipula que países } \\
\text { desenvolvidos que tenham adotado ao tratado reduzam seus níves de emissão de gases } \\
\text { do efeito estufa. Cobre dois períodos de redução de emissão: 2008-2012 e 2013-2020. } \\
\text { Trinta e sete países assinaram o tratado, com exceções como os EUA e o Canadá. O } \\
\text { tratado estabeleceu novos sistemas e créditos de carbono para países que financiam } \\
\text { programas de redução de emissões no mundo em desenvolvimento. }\end{array}$ \\
\hline $\begin{array}{l}\text { 2009, Copenhagen, UN } \\
\text { Climate Change Conference }\end{array}$ & $\begin{array}{l}\text { A desastrosa conferência foi um indicador da inabilidade de países desenvolvidos e em } \\
\text { desenvolvimento de convergirem para um consenso de como avançar nas questões de } \\
\text { mudanças climáticas. Nenhum acordo ou plano de ação foi adotado nesta conferência. }\end{array}$ \\
\hline $\begin{array}{l}\text { 2012, Rio de Janeiro, } \\
\text { Conferência ONU Rio +20 e } \\
\text { "O Futuro que Queremos" }\end{array}$ & $\begin{array}{l}\text { Muitas das discussões da Rio+20 foram acerca dos esforços fracassados de se } \\
\text { implementar algum tipo de convergência global para o desenvolvimento sustentável, } \\
\text { idelizada em 1992. O relatório “O Futuro que Queremos” apresenta este mesmo tom de } \\
\text { fracasso e argumenta em favor de uma renovação do compromisso político. }\end{array}$ \\
\hline
\end{tabular}

Fonte: adaptado de Caradonna, 2014; Waas, Hugé, Verbruggen \& Wright, 2011 
Nota-se, nos principais eventos descritos no Quadro 1, o esforço na busca do desenvolvimento sustentável. No entanto, a evolução do conceito de desenvolvimento sustentável vem apresentando diferentes abordagens, o que será discutito no próximo tópico.

\subsection{Desenvolvimento Sustentável}

A definição sugerida pela WCED (1987) para desenvolvimento sustentável é tida como um ponto de partida para a discussão atual acerca do conceito de Desenvolvimento Sustentável. Entrentanto, não existe apenas uma conceituação, pois a expressão trata de uma justaposição de dois conceitos, "Desenvolvimento" e "Sustentabilidade" que também estão em constante desenvolvimento na literatura consultada. Enquanto o primeiro termo pode ser generalizado como a busca de um estado mais avançado de alguma dimensão específica, seja ela econômica, social, institucional, ambiental, espiritual, tecnológica, etc., o segundo conceito é abordado de forma complexa e transdisciplinar (Leal Filho, 2000; Mebratu, 1998; Waas et al., 2011). Dessa forma, a conceitualização de desenvolvimento sustentável vem evoluindo de acordo com as novas descobertas e novas estruturas conceituais, por ser um campo de estudo que pode ser abordado por diversos ângulos e campos do conhecimento. O Quadro 2 apresenta algumas das diferentes abordagens para os conceitos propostos pela literatura sobre desenvolvimento sustentável:

\section{Quadro 2}

\section{Conceitos de Desenvolvimento Sustentável}

\begin{tabular}{|c|c|}
\hline Definição & Autor \\
\hline $\begin{array}{l}\text { “Desenvolvimento que atende às necessidades do presente sem comprometer a } \\
\text { capacidade das gerações futuras de atender às suas próprias necessidades" (p. 8) }\end{array}$ & (Brundtland, 1987) \\
\hline $\begin{array}{l}\text { "O desenvolvimento sustentável envolve a concepção de um sistema social e econômico, } \\
\text { que garanta que esses objetivos sejam sustentados, ou seja, que os rendimentos reais } \\
\text { e padrões educacionais aumentem, que haja melhoria da saúde da nação e que a } \\
\text { qualidade de vida geral avance." (p. 1) }\end{array}$ & $\begin{array}{l}\text { (Pearce, Markandya \& } \\
\text { Barbier, 1989) }\end{array}$ \\
\hline $\begin{array}{l}\text { "Melhorar a qualidade da vida humana dentro da capacidade de suporte dos } \\
\text { ecossistemas de apoio" (p. 10) }\end{array}$ & $\begin{array}{l}\text { (Munro, Holdgate \& } \\
\text { Prescott-Allen, 1991) }\end{array}$ \\
\hline $\begin{array}{l}\text { "A sustentabilidade é a capacidade de um sistema humano, natural ou misto, resistir ou } \\
\text { adaptar-se a mudanças endógenas ou exógenas indefinidamente"(p. 14) }\end{array}$ & (Dovers \& Handmer, 1992) \\
\hline $\begin{array}{l}\text { "A sustentabilidade é uma relação entre sistemas econômicos dinâmicos e sistemas } \\
\text { ecológicos dinâmicos maiores, mas geralmente mais lentos, em que (a) a vida humana } \\
\text { pode continuar indefinidamente, (b) os indivíduos humanos podem florescer e (c) as } \\
\text { culturas humanas podem desenvolver." (p. 8) }\end{array}$ & (Costanza, 1992) \\
\hline $\begin{array}{l}\text { "O desenvolvimento sustentável envolve a busca simultânea da prosperidade econômica, } \\
\text { da qualidade ambiental e da equidade social. " (p. 397) }\end{array}$ & (Elkington, 1997) \\
\hline $\begin{array}{l}\text { “O desenvolvimento sustentável é uma construção social, referente à evolução a longo } \\
\text { prazo de um sistema extremamente complexo - a população humana, a economia } \\
\text { incorporada nos ecossistemas e fluxo biogeoquímico do planeta." (p. 7) }\end{array}$ & (Meadows, 1998) \\
\hline $\begin{array}{l}\text { “O desenvolvimento sustentável representa uma mudança na compreensão do lugar } \\
\text { da humanidade no planeta, mas é aberto à interpretação de ser qualquer coisa, desde } \\
\text { quase sem sentido até a extrema importância para a humanidade." (p. 13) }\end{array}$ & $\begin{array}{l}\text { (Hopwood, Mellor \& } \\
\text { O'Brien, 2005) }\end{array}$ \\
\hline $\begin{array}{l}\text { “O desenvolvimento sustentável é um sistema de valores normativos, em perfeita } \\
\text { harmonia com os direitos humanos, a democracia e a liberdade (e está intimamente } \\
\text { ligado a todos esses sistemas). Assim, o desenvolvimento sustentável é essencialmente } \\
\text { um forte pronunciamento ético e moral quanto ao que deve ser feito." (p. 3) }\end{array}$ & $\begin{array}{l}\text { (Holden, Linnerud, \& } \\
\text { Banister, 2017) }\end{array}$ \\
\hline
\end{tabular}

Fonte: adaptado de Chang, Zuo, Zhao, Ziççante, Gan e Soebarto (2017) e Duran, Gogan, Artene \& Duran (2015) 
O modelo mais clássico e difundido é o do Tripé da Sustentabilidade (Elkington, 1997), no qual as ações são classificadas por três dimensões: social, ambiental e econômica. A abordagem que este modelo sugere é a reflexão de como as ações podem ser economicamente viáveis, socialmente justas e ambientalmente responsáveis.

Porém, alguns dos diversos conceitos que não são englobados nesta primeira abordagem acabam tendo influência direta no desenvolvimento, e o então detrimento destes conceitos no conjunto das dimensões do desenvolvimento sustentável resultam em uma análise incompleta. Diversos estudos se propõem a analisar as dimensões da Sustentabilidade (Ayres, 2008; Blewitt, 2008; Duran, Gogan, Artene, \& Duran, 2015; Gowdy, 2005), e com o desenvolvimento do conhecimento científico na área, a literatura adota, além das três mais difundidas, outras dimensões como: Ética e Moralidade (Aguinis \& Glavas, 2012); Política e Institucional (Acemoglu, Johnson, \& Robinson, 2005; Mavragani, Nikolaou, \& Tsagarakis, 2016; Ostrom, Schroeder, \& Wynne, 1993); Técnica (Pawłowski, 2008; Weitzman, 1997); Cultura (Blewitt, 2008; Lorek \& Spangenberg, 2014; Soini \& Birkeland, 2014); Espiritulidade (Campante \& Yanagizawa-Drott, 2015; Carroll, 2004; Rodrigues, 2010)

Os conceitos e ações que se relacionam com o Desenvolvimento Sustentável podem ser organizados hierarquicamente, como proposto por Glavic e Lukman (2007), no qual os autores utilizam as dimensões ambiental, econômica, social e política, para organizarem os conceitos de acordo com a seguinte ordem hierárquica:

- Princípios: conceitos fundamentais para o estabelecimento de um sistema mais complexo. Referem-se a apenas uma ação ou método e estão na base da hierarquia;

- Abordagens: conjunto de princípios relacionados ao mesmo tópico, construindo um sistema mais complexo. As abordagens são mais amplas que os princípios;

- Subsistemas: consiste em um conjunto de abordagens. Eles “introduzem estratégias que devem ser atendidas para alcançar a conservação integral do meio ambiente e contribuir para o bem-estar humano a curto e longo prazo. " (Glavič \& Lukman, 2007, p. 7); e

- Sistemas Sustentáveis: um sistema é um conjunto de subsistemas que trabalham em sinergia. Portanto, um sistema sustentável é o patamar mais elevado de atividades para progredir em direção ao desenvolvimento sustentável. Tais arranjos são acompanhados de uma mudança no estilo de vida e mind-set da sociedade.

\subsection{Objetivos do Desenvolvimento Sustentável}

No ano 2000, a Assembleia Geral das Nações Unidas propõe, na chamada Declaração do Milênio, os Objetivos de Desenvolvimento do Milênio (ODMs). A nova metodologia, composta por oito objetivos e vinte e duas metas mensuráveis e bem definidas, sintetizou os esforços e acordos compactuados entre diversos países. Esta nova abordagem, com indicadores quantitativos e mensuráveis, é passivel de acompanhamento, e assim se mostrou eficaz, e os ODMs obtiveram um saldo positivo (Assembly, 2000, 2015).

Em dezembro de 2015, na Agenda 2030 para o Desenvolvimento Sustentável, foram lançados os Objetivos de Desenvolvimento Sustentável (ODSs), considerados uma evolução em relação aos ODMs. Os ODSs são dezessete objetivos, desmembrados em cento e sessenta e nove metas, que são ainda mais ambiciosas por abarcarem mais dimensões do desenvolvimento sustentável (Campbell, 2017; Leal Filho, Azeiteiro, Alves, Pace, Mifsud, Brandli \& Disterheft, 2017).

O Quadro 3 mostra a evolução da visão e cooperação mundial em direção ao desenvolvimento sustentável. 
Quadro 3

ODMs e ODS

\begin{tabular}{ll}
\hline \multicolumn{1}{c}{ Objetivo de Desenvolvimento do Milênio (2000) } & \multicolumn{1}{c}{ Objetivos de Desenvolvimento Sustentável (2015) } \\
\hline 1. Acabar com a Fome e a Miséria & 1. Erradicação da Pobreza \\
2. Educação Básica de Qualidade para Todos & 2. Fome Zero \\
3. Igualdade Entre Sexos e Valorização da Mulher & 3. Boa Saúde e Bem-Estar \\
4. Reduzir a Mortalidade Infantil & 4. Educação de Qualidade \\
5. Melhorar a Saúde das Gestantes & 5. Igualdade de Gênero \\
6. Combater a Aids, Malária e Outras Doenças & 6. Água Limpa e Saneamento Básico \\
7. Qualidade de Vida e Respeito ao Meio Ambiente & 7. Energia Acessível e Limpa \\
8. Todos Trabalhando pelo Desenvolvimento & 8. Emprego Digno e Crescimento Econômico \\
& 9. Indústria, Inovação e Infraestrutura \\
& 10. Redução das Desigualdades \\
& 11. Cidades e Comunidades Sustentáveis \\
& 12. Consumo e Produção Responsáveis \\
& 13. Combate às Alterações Climáticas \\
& 14. Vida De Baixo D’Água \\
& 15. Vida Sobre a Terra \\
& 16. Paz, Justiça e Instituições Fortes \\
& 17. Parcerias em Prol das Metas
\end{tabular}

Fonte: elaborado a partir de Assembly (2000), Campbell (2017) e Mundo (2016)

A literatura atual do tema concentra-se em avaliar os indicadores existentes e desenvolver melhores indicadores, que sejam quantitativos e mais mensuráveis (Costanza, Fioramonti, Giovannini, Kubiszewski, Mortensen \& Wilkinson, 2016; Engebretsen, Heggen \& Ottersen, 2017; Hák et al., 2016; ICSU, 2015). Cada vez mais, os ODS podem ser alinhados na elaboração desses indicadores.

\section{Sustentabilidade no Mundo Corporativo}

Neste tópico, procurou-se descrever sobre os principais indicadores de sustentabilidade e de reputação empresarial que são utilizados nas pesquisas, bem como evidenciar resultados de pesquisas que relacionam práticas e indicadores de sustentabilidade com desempenho econômico-financeiro das empresas. Por fim, produrou-se discutir sobre pesquisas que mostram a evolução das formas de evidenciação da Sustentabilidade, especialmente sobre a nova proposta de disclosure pelo Relato Integrado.

\subsection{Indicadores de Sustentabilidade e Reputação Empresarial}

Ainda que haja dificuldades em uma definição única e não viesada, o mercado encontrou meios de sinalizar práticas de sustentabilidade empresarial, vinculando a Sustentabilidade à reputação empresarial, e as pesquisas mais recentes buscam entender qual o impacto da Responsabilidade Social Corporativa (RSC) na performance financeira das empresas à luz destes índices. Dentre os indicadores de Sustentabilidade e reputação empresarial, destacam-se, o Indíce de Sustentabilidade Empresarial (ISE) - BM\&FBOVESPA; o Down Jones Sustainability Indexes (DJSI); o FTSE4Good, da Bolsa de Londres; além das séries de normas internacionais ISO 14000 e 26000 e o Indice de Reputação MERCO. (ABNT, 2004; BOVESPA, 2012; Finch, 2005; Group, 2010; GVces, 2016; INDEXES, 2006; Knoepfel, 2001; Melo \& Gomes, 2007; MERCO, 2016). No Quadro 4 são evidenciados alguns dos principais índices de sustentabilidade e reputação. 
Quadro 4

Índices de Sustentabilidade

\begin{tabular}{|c|c|}
\hline Índice & Descrição \\
\hline Índice Social Domini 400 & $\begin{array}{l}\text { Composto por uma seleção de } 400 \text { companhias norte americanas das quais } 250 \\
\text { pertecem ao Índice S\&P 500. Fundado em } 1990 \text { pela Kinder, Lydenberg Domini Co. }\end{array}$ \\
\hline $\begin{array}{l}\text { Índices de Sustentabilidade Dow } \\
\text { Jones }\end{array}$ & $\begin{array}{l}\text { Calculado e analisado de uma maneira similar aos Índices Globais Dow Jones, } \\
\text { e subcaracterizado em dois índices: O ISDJ Mundo e o ISDJ STOXX, sendo o } \\
\text { último um mix de companhias europeias. Fundado em } 1999 \text { pela Dow Jones e } \\
\text { Sustainable Asset Management Group }\end{array}$ \\
\hline FTSE4Good & $\begin{array}{l}\text { Desenhado para mensurar o desempenho de companhias que demonstram } \\
\text { fortes práticas de Governança, Responsabilidade Social e Ambiental. Fundada em } \\
2001 \text { pela British Corporate Group. }\end{array}$ \\
\hline $\begin{array}{l}\text { Índice Ethibel de Sustentabilidade } \\
\text { (ESI) }\end{array}$ & $\begin{array}{l}\text { Composto por quatro índices regionais: ESI Global, ESI Americas, ESI Europa, ESI } \\
\text { Asia Pacifica. Fundado em } 2002 \text { pela Standard \& Poor's. }\end{array}$ \\
\hline $\begin{array}{l}\text { Índice de Responsabilidade Social } \\
\text { (SRI) }\end{array}$ & $\begin{array}{l}\text { Lançado na África do Sul, é considerado o primeiro índice de sustentabilidade de } \\
\text { um país emergente. Fundado em 2004, pela Bolsa de Valores de Johannesburg. }\end{array}$ \\
\hline Índice Social Calvert & $\begin{array}{l}\text { Composto por } 680 \text { companhias selecionadas de } 1000 \text { das maiores companhias } \\
\text { negociadas publicamente nos EUA. Fundado em } 2005 \text { pela Calvert Co. }\end{array}$ \\
\hline $\begin{array}{l}\text { Índice de Sustentabilidade } \\
\text { Empresarial (ISE) }\end{array}$ & $\begin{array}{l}\text { Originalmente fundado pela Corporação Financeira Internacional (IFC), o braço } \\
\text { do setor privado do Banco Mundial. É “uma ferramenta para análise comparativa } \\
\text { da performance das empresas listadas na BM\&FBOVESPA sob o aspecto da } \\
\text { sustentabilidade corporativa, baseada em eficiência econômica, equilíbrio } \\
\text { ambiental, justiça social e governança corporativa.". Foi desenhada pela GVces e } \\
\text { fundada pela BMF\&Bovespa em } 2005 \text {. }\end{array}$ \\
\hline Índice de Reputação MERCO & $\begin{array}{l}\text { O índice de Reputação MERCO é um ranking de pontuação, que iniciou em } 2004 \\
\text { e que foi construída por intermedio de uma escala de } 0 \text { a 10000, para medir } \\
\text { o conceito multidimensional da reputação corporativa, envolvendo métricas } \\
\text { de resultados econômico-financeiros, qualidade da informação, compromisso } \\
\text { da empresa com a sociedade, qualidade do produto-serviço,/respeito aos } \\
\text { direitos do consumidor, comprometimento com seus colaboradores, qualidade } \\
\text { do disclosure informacional, e ainda a qualidade tanto na gestão quanto } \\
\text { na inovação. O MERCO ranqueia a reputação das empresas atuantes no } \\
\text { conglomerado G-20 com foco nos países hispânicos. Os países em que o MERCO } \\
\text { apura os índices de reputação são: Colômbia, Chile, Argentina, Equador, Bolívia, } \\
\text { México, Peru, Alemanha, México e Brasil desde 2013. (Pinto, 2015; MERCO, 2016). }\end{array}$ \\
\hline
\end{tabular}

Fonte: elaborado a partir de Merco (2016), Orsato, Garcia, Mendes-Da-Silva, Simonetti \& Monzoni (2015) e Pinto, Freire \& Dos Santos (2015).

Na literatura, verificam-se pesquisas que buscam determinantes para a participação de empresas em índices de sustentabilidade e para divulgação de informações sociais, por meio de demonstrações financeiras. Dessa forma, alguns estudos encontram evidências de que as características das empresas podem influenciar na inclusão em índices de Sustentabilidade (fonte, ano; fonte, ano). Por exemplo, resultados sugerem que empresas com maior potencial de crescimento apresentam Responsabilidade Social Corporativa (RSC) mais elevada, pois essas têm mais oportunidade de adotar ações sustentáveis em suas operações (Artiach, Lee, Nelson \& Walker, 2010; Belkaoui \& Karpik, 1989; Nunes, Teixeira, Nossa \& Galdi, 2010). Outro resultado que se mostra robusto, mesmo ao analisar diferentes empresas, índices, períodos de tempo e métodos, é o de que o tamanho da empresa pode influenciar na dua decisão por compor os índices de sustentabilidade. $\mathrm{O}$ argumento é de que empresas maiores estão mais sujeitas ao escrutínio dos stakeholders, sendo uma decisão estratégica sinalizar responsabilidade social corparativa e preocupação com o desenvolvimento sustentável. Assim, um importante fator que leva as empresas a terem iniciativas voluntárias de Sustentabilidade é o valor intangível criado por tais ações, como acesso a novos conhecimentos, inovação, e ganhos de reputação (Andrade, Bressan, Iquiapaza \& de Melo Moreira, 2013; Artiach et al., 2010; Nunes et al., 2010; Orsato et al., 2015). 


\subsection{Sustentabilidade e desempenho econômico-financeiro das empresas}

Embora já existisse alguma discussão acerca da relação entre empresas e sociedade, o conceito de RSC é relativamente novo na literatura, e muitos autores consideram a obra Social Responsibilities of the Businessman (1953), de Howard Bowen, como a primeira teorização do assunto, ao propor que os homens de negócios deveriam tomar decisões e seguir diretrizes que vão ao encontro dos objetivos e valores da sociedade que compõem, sendo esta linha argumentativa o foco principal da literatura nos anos de 1950 e 1960 (Bowen, 1953; Carroll, 1979; Lee, 2008; Wartick \& Cochran, 1985).

No período de 1970 a 1980, aproximadamente, o interesse próprio dos agentes econômicos tinha a única responsabilidade social da empresa relacionada ao aumento do lucro para os acionistas. Nos anos 1980, foi desenvolvido um primeiro modelo que propunha quatro categorias de reponsabilidade social (Econômica, Legal, Ética e Filantrópica), como um caminho natural para responder às críticas referentes à falta de rigor analítico. Nos anos 1990, a teoria do Stakeholder, que aborda as pressões sofridas pelas empresas por diversos grupos sociais e a RSC como parte da gestão estratégica da empresa ganham protagonismo (Carroll, 1979; Edward, 1984; Freguete, Nossa \& Funchal, 2015; Friedman, 1970; Jones, 1995; Lee, 2008; Matten \& Moon, 2008; Wartick \& Cochran, 1985).

Apesar desta evolução, as divergências ainda se fazem presentes. Um estudo realizado por Dahlsrud (2008) se propôs a analisar as diferentes conceituações de RSC utilizadas na literatura. O autor identificou 37 definições. Ao comparar suas diferenças e semelhanças, construiu uma classificação contendo as seguintes dimensões de responsabilidades das ações da firma: Stakeholders, Sociedade, Economia, Meio Ambiente e Voluntariado (Dahlsrud, 2008).

Ao propor que as empresas se encontram em um contexto no qual sofrem pressões de diversos atores sociais, a teoria dos Stakeholders prevê que o exercício da RSC por parte dessas empresas pode influenciar positivamente em melhores resultados financeiros das empresas que a exercem, qualificados por normas técnicas e índices.

Os resultados empíricos acerca dos custos e benefícios de se participar de índices como os citados ainda são contraditórios, não havendo consenso na literatura. Diversos estudos sugerem que, em curto prazo, a presença, ou não, das empresas nos índices de sustentabilidade não indicam maior retorno financeiro (Bansal \& Hunter, 2003; Darnall, 2006; Delmas, 2002; Delmas \& Montes-Sancho, 2011; Gilbert \& Rasche, 2007; King \& Lenox, 2001; King, Lenox \& Terlaak, 2005; Russo \& Fouts, 1997). Muitos desses estudos concluem em suas pesquisas que não encontraram relação alguma entre sustentabilidade e desempenho financeiro ou percepção positiva de acionistas sobre o preço das ações. Esses autores destacam ainda que as empresas buscam divulgar que estão comprometidos com certificação ou participam de índices de sustentabilidade ou ainda de reputação devido às forças externas. Assim, as empresas investem em sustentabilidade não somente porque isto traz resultados financeiros, mas, sim, por uma questão de sobrevivência, a depender da área de atuação, devido às pressões feitas por clientes, fornecedores e outras partes interessadas no processo, a legitimidade da certificação e as capacidades internas envolvidas.

Apesar de existirem algumas evidências de que a sustentabilidade não tem correlação positiva com melhor performance financeira, como já apontado em outros estudos, existem pesquisas que encontraram relação positiva entre sustentabilidade e desempenho financeiro e a percepção dos mercados acionários sobre a sustentabilidade e os preços das ações (Allouche \& Laroche, 2005; Arimura, Hibiki \& Katayama, 2008; Darnall \& Edwards, 2006; Darnall, Jolley \& Ytterhus, 2007; Endrikat, Guenther \& Hoppe, 2014; Heras-Saizarbitoria, Molina-Azorín \& Dick, 2011; Lu, Chau, Wang \& Pan, 2014; Melnyk, Sroufe \& Calantone, 2003; Montabon, Melnyk, Sroufe \& Calantone, 2000; Nakano \& Managi, 2006; Orlitzky, Schmidt \& Rynes, 2003; Perez, Amichai-Hamburger \& Shterental, 2009; Potoski \& Prakash, 2013; Zhao, 2008).

Os trabalhos que corroboram a tese de relação positiva entre sustentabilidade e desempenho financeiro sugerem que a relação entre as variáveis se mostra bidirecional e simultânea. Ou seja, o bom desempenho financeiro da firma sinaliza aos investidores uma boa oportunidade, disponibilizando às empresas 
mais capacidade de melhorarem seu desempenho ambiental e social. Consequentemente, a diminuição dos custos de agência e a redução da assimetria informacional influenciados pelas ações de RSC impactam positivamente o desempenho financeiro e a percepção dos acionistas sobre o mercado acionário (Cheng, Ioannou \& Serafeim, 2014; Jo \& Harjoto, 2012; Luo, Wang, Raithel \& Zheng, 2015; Mishra \& Suar, 2010; Orlitzky et al., 2003; Preston \& O’bannon, 1997; Scholtens, 2008).

Entretanto, a complexidade de se conceituar a sustentabilidade implica uma dificuldade em definir e mensurar os indicadores deste conceito, o que é um ponto contundente de crítica na literatura. Destaca-se que a forma de divulgação da sustentabilidade por meio do Balanço Social, GRI, Site das empresas, Demonstrações Contábeis, Relato Integrado, etc. pode influenciar na percepção dos stakeholders sobre a sustentabilidade e sobre o desempenho da empresa. Verificou-se na literatura que diferentes fontes e informações foram utilizadas para mensurar Sustentabilidade. Verificou-se ainda que diferentes escopos metodológicos foram utilizados, e sabe-se que variadas metodologias podem influenciar o resultado das pesquisas, devido muitas vezes à ausência de variáveis relevantes na estimação e parâmetros unificados. Além da complexidade do fenômeno, a teoria é pouco consistente e vaga. Tais características fazem com que a metodologia utilizada também seja fator influenciador na relação entre as variáveis (Borba, 2005; Korhonen, 2003; Van Beurden \& Gössling, 2008). Apesar de alguns resultados de pesquisas encontrarem evidências empíricas de que não há relação da sustentabilidade com indicadores econômico-financeiros, registra-se que a iniciativa de uma instituição em se preocupar com a sustentabilidade já é um fator positivo para sua continuidade. De qualquer forma, percebe-se que há espaço para novas pesquisas mais robustas com teorias de base mais consistentes.

\subsection{Relato Integrado: nova forma de evidenciação contábil}

O Global Reporting Iniciative (GRI) vem sendo considerado o modelo de relatório de sustentabilidade mais disseminado no mundo corporativo. O GRI iniciou esse movimento em 1998 e tinha como missão "elaborar e difundir as Diretrizes para a elaboração de relatórios de sustentabilidade aplicáveis global e voluntariamente pelas organizações que desejassem dar informações sobre os aspectos econômicos, ambientais e sociais de suas atividades, produtos e serviços" (GRI, 2000, p. 3). Ao longo dos anos, o modelo de relatório e as diretrizes do GRI foram evoluindo e contribuíram para a melhoria da orientação para o desenvolvimento de relatórios de sustentabilidade pelas empresas (GRI, 2000).

Em 2010, um novo debate internacional se iniciou em torno de uma nova forma de se fazer a evidenciação da Sustentabilidade e que foi intitulado de Relato Integrado (IR - do inglês Integrated Reporting). A iniciativa vem do International Integrated Reporting Council (IIRC), que reúne membros de vários países (inclusive o GRI) e é liderado por Sua Alteza Real, o Príncipe de Gales.

Os elementos de conteúdo do Relato Integrado têm os seguintes princípios inovadores, segundo Mio (2016, p. 5): foco estratégico e orientação futura; conectividade de informação; relacionamentos de partes interessadas; materialidade; concisão; responsabilidade e integridade; consistência e comparabilidade; visão organizacional e ambiente externo; governança; modelo de negócio; riscos e oportunidades; estratégia e alocação de recursos; desempenho; perspectiva e base de preparação e apresentação. Ainda segundo Mio (2016), a ideia do Relato Integrado é norteada na integração baseada em um processo que se inicia a partir da estratégia e reestrutura todo o sistema de divulgação corporativa, de acordo com uma perspectiva de pensamento integrada

Haller (2016) destaca que o principal conceito do Relato Integrado é a criação de valor gerado aos diversos capitais (financeiro, manufaturado, intelectual, humano, natural, social e de relacionamento) evidenciado para os stakeholders, shareholders e outros usuários.

Algumas empresas ao redor do mundo foram convidadas pelo IIRC a iniciarem o desenvolvimento do Relato Integrado. No Brasil, Ricardino Filho \& Carvalho (2016) apresentam os casos do Itaú Unibanco e da Natura como os primeiros a adotarem esse novo formato de relato. 
Com a adoção do Relato Integrado, há a expectativa de que se reduza o custo de capital das empresas. Carvalho \& Murcia (2016, p. 35) argumentam que a redução do custo da dívida em médio e longo prazos podem resultar de dois fatores: “(i) a adoção de um modelo de negócio sustentável devido ao pensamento integrado e (ii) uma redução na assimetria de informação causada por maior transparência, permitindo previsões mais acuradas, ambos levando a retornos positivos para investidores e credores no longo prazo".

Com relação às pesquisas sobre Relato Integrado, ainda se encontram em estágio incipiente, considerando a recente criação do modelo e ainda carece de robustez em sua estrutura conceitual. Dumay, Bernardi, Guthrie \& Demartini (2016) concluem em seu estudo que, embora pareça cedo para se fazer pesquisa sobre esse tema, ela é muito bem-vinda, especialmente porque o tema está em um estágio no qual deve haver um debate sobre a possibilidade de harmonização de conceitos, que pode depender da criação de comunidades internacionais que reúnam profissionais, formuladores de políticas e líderes de pensamento ao redor o mundo, como aconteceu no projeto GRI nas últimas duas décadas. Dumay et al. (2016) destacam ainda que a maioria das pesquisas sobre Relato Integrado é de natureza normativa, e isso pode ser encarado como natural, considerando os diferentes estágios em que uma pesquisa evolui. E isso pode ser um ponto de partida para se desenvolver a compreensão sobre o impacto dessas novas tecnologias na prática por meio de futuras pesquisas empíricas.

Ainda sobre os desafios do Relato Integrado na prática, de Villiers, Rinaldi, \& Unerman (2014) argumentam que o rápido desenvolvimento da política integrada de relatórios e o início do desenvolvimento na prática apresentam desafios teóricos e empíricos devido às diferentes formas em que os relatos integrados são entendidos e promulgados nas instituições. O autor assinala ainda que isso abre espaço para novas pesquisas acadêmicas para orientar a evolução da política e da prática.

No campo da auditoria do Relato Integrado, a pesquisa de Oprisor (2015) concluiu que é difícil de se obter um nível elevado (ou razoável) de segurança no caso de relatórios integrados diante da falta de regulamentos de auditoria, a característica específica da empresa de relatórios integrados e indicadores-chave de desempenho para informações não financeiras. Essas são também lacunas para o desenvolvimento de pesquisas sobre o tema.

\section{Considerações Finais}

A ênfase no conceito de desenvolvimento sustentável iniciou-se mais fortemente nos anos 1980 e 1990 , especialmente no que se refere às questões sociais e ambientais, chegando atualmente aos Objetivos do Desenvolvimento Sustentável (ODS) propostos pela ONU. Isso provocou um aumento da pressão da sociedade organizada, especialmente em relação ao mundo corporativo. Assim, as empresas passaram a incorporar conceitos relacionados à Sustentabilidade que antes não faziam parte de suas operações.

Nesse sentido, iniciou-se um processo de criação de indicadores de Sustentabilidade com vistas a dar sinalização ao mercado referente às empresas que incorporaram conceitos de Sustentabilidade em suas práticas. A partir daí pesquisadores começaram a desenvolver pesquisas relacionando práticas e indicadores de Sustentabilidade com desempenho econômico-financeiro das empresas. O resultado dessas pesquisas tem seguido diferentes direcionamentos, muitas vezes controversos, em alguns casos, por adotarem diferentes metodologias.

A forma de evidenciação da Sustentabilidade também vem evoluindo ao longo dos anos. O debate atual está em torno do Relato Integrado que tem o objetivo de relatar informações de forma integrada e que apresente agregação de valor aos seus usuários e, principalmente, buscam reduzir a assimetria de informação em relação à Sustentabilidade dos negócios.

Nota-se, assim, que são muitas as lacunas no tema Sustentabilidade que ainda necessitam de avanço nas pesquisas, especialmente em relação à sua mensuração e evidenciação e seus relacionamentos com o desempenho das empresas. 
Ressalta-se, por fim, algumas limitações desta revisão da literatura, considerando que a ideia foi descrever a evolução do conceito de Sustentabilidade e algumas interfaces com a Contabilidade, sem a pretensão de realizar uma análise critíca de toda a literatura de Sustentabilidade e Contabilidade com todos os seus criteriosos aspectos metodológicos.

\section{Referências}

ABNT, N. B. R. (2004). 16001. Associação Brasileira de normas Técnicas. Responsabilidade Social-sistema de Gestão-requisitos. Recuperado em 15 de novembro, 2017, de http://www.inmetro.gov.br/qualidade/responsabilidade_social/norma_nacional.asp.

Acemoglu, D., Johnson, S., \& Robinson, J. A. (2005). Institutions as a fundamental cause of long-run growth. Handbook of Economic Growth, 1, 385-472. https://economics.mit.edu/files/4469

Aguinis, H., \& Glavas, A. (2012). What We Know and Don't Know About Corporate Social Responsibility. Journal of Management, 38(4), 932-968. https://doi.org/10.1177/0149206311436079

Allen, C., Metternicht, G., \& Wiedmann, T. (2016). National pathways to the Sustainable Development Goals (SDGs): A comparative review of scenario modelling tools. Environmental Science and Policy, 66, 199-207. https://doi.org/10.1016/j.envsci.2016.09.008

Allouche, J., \& Laroche, P. (2005). A meta-analytical investigation of the relationship between corporate social and financial performance. Revue de Gestion Des Ressources Humaines, (57), 18.

Andrade, L. P., Bressan, A. A., Iquiapaza, R. A., \& de Melo Moreira, B. C. (2013). Determinantes de adesão ao Índice de Sustentabilidade Empresarial da BM\&FBOVESPA e sua relação com o valor da empresa. Revista Brasileira de Finanças, 11(2).

Arimura, T. H., Hibiki, A., \& Katayama, H. (2008). Is a voluntary approach an effective environmental policy instrument?: A case for environmental management systems. Journal of Environmental Economics and Management, 55(3), 281-295.

Arrow, K., Dasgupta, P., Goulder, L., Daily, G., Ehrlich, P., Heal, G., ... Summer, N. (2004). Are We Consuming Too Much ? The Journal of Economic Perspective, 18(3), 147-72. https://doi. org/10.1257/0895330042162377

Artiach, T., Lee, D., Nelson, D., \& Walker, J. (2010). The determinants of corporate sustainability performance. Accounting \& Finance, 50(1), 31-51.

Assembly, U. N. G. (1982). World Charter for Nature. United Nations General Assembly Resolution, 37(7).

Assembly, U. N. G. (2000). United Nations millennium declaration. United Nations General Assembly.

Assembly, U. N. G. (2015). Draft Outcome Document of the United Nations Summit for the Adoption of the Post-2015 Development Agenda.

Ayres, R. U. (2008). Sustainability economics: Where do we stand? Ecological Economics, 67(2), 281-310. https://doi.org/10.1016/j.ecolecon.2007.12.009

Bansal, P., \& Hunter, T. (2003). Strategic explanations for the early adoption of ISO 14001. Journal of Business Ethics, 46(3), 289-299.

Belkaoui, A., \& Karpik, P. G. (1989). Determinants of the corporate decision to disclose social information. Accounting, Auditing \& Accountability Journal, 2(1).

Bithas, K. (2011). Sustainability and externalities: Is the internalization of externalities a sufficient condition for sustainability? Ecological Economics, 70(10), 1703-1706. https://doi.org/10.1016/j.ecolecon.2011.05.014 
Blewitt, J. (2008a). Community, empowerment and sustainable development. Green books.

Blewitt, J. (2008b). Understanding sustainable development. Vasa. https://doi.org/10.2139/ssrn.956240

Borba, P. da R. F. (2005). Relação entre desempenho social corporativo e desempenho financeiro de empresas no Brasil. Universidade de São Paulo.

BOVESPA, B. M. F. (2012). Índice de Sustentabilidade empresarial (ISE). Arquivo Eletrônico. São Paulo. Recuperado em 15 de novembro, 2017, de http://www.bmfbovespa.com.br/pt_br/produtos/indices/indices-de-sustentabilidade/indice-de-sustentabilidade-empresarial-ise.htm.

Bowen, H. R. (1953). Social responsibilities of the businessman. Harper.

Brandt, P., Ernst, A., Gralla, F., Luederitz, C., Lang, D. J., Newig, J., ... Von Wehrden, H. (2013). A review of transdisciplinary research in sustainability science. Ecological Economics, 92. https://doi. org/10.1016/j.ecolecon.2013.04.008

Brundtland, G. (1987). Report of the World Commision on Environement and Development: Our Common Future. Oxford Paperbacks, Report of, 400. https://doi.org/10.2307/2621529

Campante, F., \& Yanagizawa-Drott, D. (2015). Does religion affect economic growth and happiness? Evidence from Ramadan. The Quarterly Journal of Economics, 130(2), 615-658.

Campbell, D. A. (2017). An Update on the United Nations Millennium Development Goals. Journal of Obstetric, Gynecologic \& Neonatal Nursing, 46(3), e48-e55. https://doi.org/https://doi.org/10.1016/j. jogn.2016.11.010

Caniglia, G., Schäpke, N., Lang, D. J., Abson, D. J., Luederitz, C., Wiek, A., ... von Wehrden, H. (2017). Experiments and evidence in sustainability science: A typology. Journal of Cleaner Production. https://doi.org/10.1016/j.jclepro.2017.05.164

Caradonna, J. L. (2014). Sustainability: A history. Oxford University Press.

Carlowitz, H. Von, \& Rohr, J. von. (1732). Sylvicultura oeconomica. Retrieved from https://books.google. com.br/books?hl=pt-BR\&lr=\&id=bHJDAAAAcAAJ\&oi=fnd\&pg=PA36\&dq=Sylvicultura+Oeco nomica\&ots=JBBomcnt_1\&sig=8BrluhAp9vwQ0M8QeGMWRD4VSgU

Carroll, A. B. (1979). A three-dimensional conceptual model of corporate performance. Academy of Management Review, 4(4), 497-505.

Carroll, J. E. (2004). Sustainability and spirituality.

Carvalho, N., \& Murcia, F. D.-R. (2016). The Relationship Between Integrated Reporting and Cost of Capital. In Integrated Reporting (pp. 253-268). Springer.

Chang, R. D., Zuo, J., Zhao, Z. Y., Zillante, G., Gan, X. L., \& Soebarto, V. (2017). Evolving theories of sustainability and firms: History, future directions and implications for renewable energy research. Renewable and Sustainable Energy Reviews, 72(January), 48-56. https://doi.org/10.1016/j. rser.2017.01.029

Cheng, B., Ioannou, I., \& Serafeim, G. (2014). Corporate social responsibility and access to finance. Strategic Management Journal, 35(1), 1-23.

Clark, B., \& Foster, J. B. (2001). William Stanley Jevons and The Coal Question An Introduction to Jevons's "Of the Economy of Fuel." Organization \& Environment, 14(1), 93-98.

Costanza, R. (1989). What is ecological economics? Ecological Economics, 1(1), 1-7.

Costanza, R. (1992). Ecological economics: the science and management of sustainability. Columbia University Press.

Costanza, R., Cumberland, J. H., Daly, H., Goodland, R., Norgaard, R. B., Kubiszewski, I., \& Franco, C. (2014). An introduction to ecological economics. CRC Press. 
Costanza, R., Daly, L., Fioramonti, L., Giovannini, E., Kubiszewski, I., Mortensen, L. F., ... Wilkinson, R. (2016). Modelling and measuring sustainable wellbeing in connection with the UN Sustainable Development Goals. Ecological Economics, 130, 350-355. https://doi.org/10.1016/j.ecolecon.2016.07.009

Cropper, M. L., \& Oates, W. E. (1992). Environmental economics: a survey. Journal of Economic Literature, 30(2), 675-740.

Dahlsrud, A. (2008). How corporate social responsibility is defined: an analysis of 37 definitions. Corporate Social Responsibility and Environmental Management, 15(1), 1-13.

Darnall, N. (2006). Why firms mandate ISO 14001 certification. Business \& Society, 45(3), 354-381.

Darnall, N., \& Edwards, D. (2006). Predicting the cost of environmental management system adoption: the role of capabilities, resources and ownership structure. Strategic Management Journal, 27(4), 301-320.

Darnall, N., Jolley, G. J., \& Ytterhus, B. (2007). Understanding the relationship between a facility's environmental and financial performance. Environmental Policy and Corporate Behaviour, 213-259.

de Villiers, C., Rinaldi, L., \& Unerman, J. (2014). Integrated Reporting: Insights, gaps and an agenda for future research. Accounting, Auditing \& Accountability Journal, 27(7), 1042-1067.

de Vries, B. J. M., \& Petersen, A. C. (2009). Conceptualizing sustainable development. An assessment methodology connecting values, knowledge, worldviews and scenarios. Ecological Economics, 68(4), 1006-1019. https://doi.org/10.1016/j.ecolecon.2008.11.015

Delmas, M. A. (2002). The diffusion of environmental management standards in Europe and in the United States: An institutional perspective. Policy Sciences, 35(1), 91-119.

Delmas, M. A., \& Montes-Sancho, M. J. (2011). An institutional perspective on the diffusion of international management system standards: The case of the environmental management standard ISO 14001. Business Ethics Quarterly, 21(1), 103-132.

Dovers, S. R., \& Handmer, J. W. (1992). Uncertainty, sustainability and change. Global Environmental Change, 2(4), 262-276.

Du Pisani, J. A. (2006). Sustainable development - historical roots of the concept. Environmental Sciences, 3(2), 83-96. https://doi.org/10.1080/15693430600688831

Dumay, J., Bernardi, C., Guthrie, J., \& Demartini, P. (2016). Integrated reporting: a structured literature review. In Accounting Forum (Vol. 40, pp. 166-185). Elsevier.

Duran, D. C., Gogan, L. M., Artene, A., \& Duran, V. (2015). The Components of Sustainable Development - A Possible Approach. Procedia Economics and Finance, 26(15), 806-811. https://doi.org/10.1016/ S2212-5671(15)00849-7

Edward, F. R. (1984). Strategic Management: A stakeholder approach. Boston: Pitman, 46.

Elkington, J. (1997). Cannibals with forks. The Triple Bottom Line of 21st Century, 73.

Endrikat, J., Guenther, E., \& Hoppe, H. (2014). Making sense of conflicting empirical findings: A meta-analytic review of the relationship between corporate environmental and financial performance. European Management Journal, 32(5), 735-751.

Engebretsen, E., Heggen, K., \& Ottersen, O. P. (2017). The Sustainable Development Goals: ambiguities of accountability. International Organization, 108, 396-405.

Field, B. C., \& Field, M. K. (1997). Environmental economics: an introduction. Sustainable Human Development Review, 105. 
Filho, W. L. (2000). Dealing with misconceptions on the concept of sustainability. International Journal of Sustainability in Higher Education, 1(1), 9-19. https://doi.org/10.1108/1467630010307066

Finch, N. (2005). The emergence of CSR and sustainability indices.

Freguete, L. M., Nossa, V., \& Funchal, B. (2015). Responsabilidade Social Corporativa e Desempenho Financeiro das Empresas Brasileiras na Crise de 2008/Corporate Social Responsibility and Brazilian Firms' Financial Performance. Revista de Administração Contemporânea, 19(2), 232.

Friedman, M. (1970). The Social Responsibility of Business is to Increase its Profits.

Gerlagh, R. (2017). Generous Sustainability. Ecological Economics, 136, 94-100. https://doi.org/10.1016/j. ecolecon.2017.02.012

Gilbert, D. U., \& Rasche, A. (2007). Discourse ethics and social accountability: The ethics of SA 8000. Business Ethics Quarterly, 17(2), 187-216.

Glavič, P., \& Lukman, R. (2007). Review of sustainability terms and their definitions. Journal of Cleaner Production. Retrieved from http://www.sciencedirect.com/science/article/pii/S0959652607000029

Goodland, R. (1995). The Concept of Environmental Sustainability. Annual Review of Ecology and Systematics, 26(1), 1-24. https://doi.org/10.1146/annurev.es.26.110195.000245

Goodland, R., \& Ledec, G. (1987). Neoclassical economics and principles of sustainable development. Ecological Modelling, 38(1-2), 19-46. https://doi.org/10.1016/0304-3800(87)90043-3

Gowdy, J. (2005). Toward a new welfare economics for sustainability. Ecological Economics, 53(2), 211-222. https://doi.org/10.1016/j.ecolecon.2004.08.007

GRI. (2000). GRI - Global Reporting Iniciative.

Griggs, D., Stafford-Smith, M., Gaffney, O., Rockström, J., Ohman, M. C., Shyamsundar, P., ... Noble, I. (2013). Policy: Sustainable development goals for people and planet. Nature, 495(7441), 305-7. https://doi.org/10.1038/495305a

Grober, U. (2007). Deep roots-. A Conceptual History Of "sustainable development"(Nachhaltigkeit), Wissenschaftszentrum Berlin Fur Sozialforschung, 8-9.

Group, F. (2010). FTSE4Good Index series inclusion criteria. London: FTSE Group.

GVces. (2016). O que é ISE? Recuperado em 7 de outubro, 2017, de http://isebvmf.com.br/o-que-e-oise?locale $=$ pt-br.

Hák, T., Janoušková, S., \& Moldan, B. (2016). Sustainable Development Goals: A need for relevant indicators. Ecological Indicators, 60, 565-573.

Haller, A. (2016). Value Creation: A Core Concept of Integrated Reporting. In Integrated Reporting (pp. 37-57). Springer.

Hellwig, K. (2005). Sustainability revisited. Economics Letters, 87(2), 193-197. https://doi.org/10.1016/j. econlet.2004.10.013

Heras-Saizarbitoria, I., Molina-Azorín, J. F., \& Dick, G. P. M. (2011). ISO 14001 certification and financial performance: selection-effect versus treatment-effect. Journal of Cleaner Production, 19(1), 1-12.

Holden, E., Linnerud, K., \& Banister, D. (2017). The Imperatives of Sustainable Development. Sustainable Development, 25(3), 213-226. https://doi.org/10.1002/sd.1647

Hopwood, B., Mellor, M., \& O’Brien, G. (2005). Sustainable development: mapping different approaches. Sustainable Development, 13(1), 38-52. 
ICSU, I. (2015). Review of the sustainable development goals: The science perspective. Paris: International Council for Science (ICSU). Recuperado em 15 de novembro, 2017, de https://www.icsu.org/ publications/reports-and-reviews/review-of-targets-for-the-sustainable-development-goals-the-science-perspective-2015.

Illge, L., \& Schwarze, R. (2009). A matter of opinion-How ecological and neoclassical environmental economists and think about sustainability and economics. Ecological Economics, 68(3), 594-604. https://doi.org/10.1016/j.ecolecon.2008.08.010

INDEXES, D. J. S. (2006). Indexes. Recuperado em 22 de outubro, 2017, de http://www. sustainability-indexes. com>.

Jerneck, A., Olsson, L., Ness, B., Anderberg, S., Baier, M., Clark, E., ... Persson, J. (2011). Structuring sustainability science. Sustainability Science, 6(1), 69-82. https://doi.org/10.1007/s11625-010-0117-x

Jevons, W. S. (1866). The coal question: an enquiry concerning the progress of the Nation, and the probable exhaustion of our coal-mines. Macmillan.

Jo, H., \& Harjoto, M. A. (2012). The causal effect of corporate governance on corporate social responsibility. Journal of Business Ethics, 106(1), 53-72.

Jones, T. M. (1995). Instrumental stakeholder theory: A synthesis of ethics and economics. Academy of Management Review, 20(2), 404-437.

Kajikawa, Y. (2008). Research core and framework of sustainability science. Sustainability Science, 3(2), 215-239. https://doi.org/10.1007/s11625-008-0053-1

King, A. A., \& Lenox, M. J. (2001). WHO ADOPTS MANAGEMENT STANDARDS EARLY? AN EXAMINATION OF ISO 14001 CERTIFICATIONS. In Academy of Management Proceedings (Vol. 2001, pp. A1-A6). Academy of Management.

King, A. A., Lenox, M. J., \& Terlaak, A. (2005). The strategic use of decentralized institutions: Exploring certification with the ISO 14001 management standard. Academy of Management Journal, 48(6), 1091-1106.

Klöpffer, W. (2003). Life-Cycle based methods for sustainable product development. The International Journal of Life Cycle Assessment. Retrieved from http://search.proquest.com/openview/e0b94b6bd af6c3f94c0da618e273954c/1?pq-origsite $=$ gscholar\&cbl=54409

Knoepfel, I. (2001). Dow Jones sustainability group index: A global benchmark for corporate sustainability. Corporate Environmental Strategy, 8(1), 6-15.

Komiyama, H., \& Takeuchi, K. (2006). Sustainability science: building a new discipline. Springer.

Kopnina, H. (2015). The victims of unsustainability: a challenge to sustainable development goals. International Journal of Sustainable Development \& World Ecology, 4509(November), 1-9. https://doi. org/10.1080/13504509.2015.1111269

Korhonen, J. (2003). Should we measure corporate social responsibility? Corporate Social Responsibility and Environmental Management, 10(1), 25-39.

Leal Filho, W., Azeiteiro, U., Alves, F., Pace, P., Mifsud, M., Brandli, L., ... Disterheft, A. (2017). Reinvigorating the sustainable development research agenda: the role of the sustainable development goals (SDG). International Journal of Sustainable Development \& World Ecology, 0(0), 1-12. https://doi. org/10.1080/13504509.2017.1342103

Lee, M. P. (2008). A review of the theories of corporate social responsibility: Its evolutionary path and the road ahead. International Journal of Management Reviews, 10(1), 53-73. 
Lorek, S., \& Spangenberg, J. H. (2014). Sustainable consumption within a sustainable economy - Beyond green growth and green economies. Journal of Cleaner Production, 63, 33-44. https://doi. org/10.1016/j.jclepro.2013.08.045

Lu, W., Chau, K. W., Wang, H., \& Pan, W. (2014). A decade's debate on the nexus between corporate social and corporate financial performance: a critical review of empirical studies 2002-2011. Journal of Cleaner Production, 79, 195-206.

Luo, X., Wang, H., Raithel, S., \& Zheng, Q. (2015). Corporate social performance, analyst stock recommendations, and firm future returns. Strategic Management Journal, 36(1), 123-136.

Malthus, T. R. (1798). An Essay on the Principle of Population, as it Affects the Future Imporvement of Society, with Remarks on the Speculations of Mr. Godwin, M. Condorcet, and Other Writers. The Lawbook Exchange, Ltd.

Marsh, G. P. (1965). Man and nature. University of Washington Press.

Marshall, J. D., \& Toffel, M. W. (2005). Framing the elusive concept of sustainability: A sustainability hierarchy. Environmental Science and Technology, 39(3), 673-682. https://doi.org/10.1021/es040394k

Matten, D., \& Moon, J. (2008). "Implicit" and "explicit" CSR: a conceptual frame- work for a comparative understanding of corporate social responsibility. Academy of Management Review, 33(2), 404-424. https://doi.org/10.5465/AMR.2008.31193458

Mavragani, A., Nikolaou, I., \& Tsagarakis, K. (2016). Open Economy, Institutional Quality, and Environmental Performance: A Macroeconomic Approach. Sustainability, 8(7), 601. https://doi.org/10.3390/su8070601

Meadows, D. H. (1998). Indicators and information systems for sustainable development.

Mebratu, D. (1998). Sustainability and sustainable development: Historical and conceptual review. Environmental Impact Assessment Review, 18(6), 493-520. https://doi.org/10.1016/S0195-9255(98)00019-5

Melnyk, S. A., Sroufe, R. P., \& Calantone, R. (2003). Assessing the impact of environmental management systems on corporate and environmental performance. Journal of Operations Management, 21(3), 329-351.

Melo, C. M., \& Gomes, E. R. (2007). NBR 16001: a norma brasileira de gestão da responsabilidade social.

MERCO. (2016). Metodologia e informe de Verificacion Merco Empresa Brasil.

Mikhailova, I. (2004). Sustentabilidade : Evolução Dos Conceitos Teóricos E Os Problemas Da Mensuração Prática. Revista Economia E Desenvolvimento, 16(n¹6), 22-41.

Mill, J. S. (1848). Of the stationary state. Principles of Political Economy Book IV: Influence of the Progress of Society.

Miller, T. R., Wiek, A., Sarewitz, D., Robinson, J., Olsson, L., Kriebel, D., \& Loorbach, D. (2014). The future of sustainability science: a solutions-oriented research agenda. Sustainability science, 9(2), 239246.. https://doi.org/10.1007/s11625-013-0224-6

Mio, C. (2016). Integrated Reporting: A New Accounting Disclosure. Springer.

Mishra, S., \& Suar, D. (2010). Does corporate social responsibility influence firm performance of Indian companies? Journal of Business Ethics, 95(4), 571-601.

Montabon, F., Melnyk, S. A., Sroufe, R., \& Calantone, R. J. (2000). ISO 14000: assessing its perceived impact on corporate performance. Journal of Supply Chain Management, 36(1), 4-16.

Mundo, T. N. (2016). A Agenda 2030 para o Desenvolvimento Sustentável. Recuperado em 15 de novembro, 2017, de http://artigo19.org/wp-content/uploads/2015/09/ods_transforming_our_world_versc3a3o-final-pt.docx. 
Munro, D. A., Holdgate, M. W., \& Prescott-Allen, R. (1991). Caring for the earth: a strategy for sustainable living; summary. International Union for Conservation of Nature and Natural Resources, Gland, $\mathrm{CH}$.

Nakano, M., \& Managi, S. (2006). The effect of ISO 14001 adoption on firm's productivity. Kankyo Kagaku Kai Shi, 19(5), 385-395.

Nunes, J. G., Teixeira, A. J. C., Nossa, V., \& Galdi, F. C. (2010). Análise das variáveis que influenciam a adesão das empresas ao índice BM\&F Bovespa de sustentabilidade empresarial. Revista Base (Administração E Contabilidade) Da UNISINOS, 7(4).

Orlitzky, M., Schmidt, F. L., \& Rynes, S. L. (2003). Corporate social and financial performance: A meta-analysis. Organization Studies, 24(3), 403-441.

Orsato, R. J., Garcia, A., Mendes-Da-Silva, W., Simonetti, R., \& Monzoni, M. (2015). Sustainability indexes: why join in? A study of the "Corporate Sustainability Index (ISE)"in Brazil. Journal of Cleaner Production, 96, 161e170.

Ostrom, E., Schroeder, L., \& Wynne, S. (1993). Institutional incentives and sustainable development: infrastructure policies in perspective. Westview Press.

Pádua, J. A. (2010). As bases teóricas da história ambiental. Estudos Avançados, 24(68), 81-101.

Pappas, E., Pappas, J., \& Sweeney, D. (2015). Walking the walk: Conceptual foundations of the Sustainable Personality. Journal of Cleaner Production, 86, 323-334. https://doi.org/10.1016/j.jclepro.2014.08.077

Pawłowski, A. (2008). How many dimensions does sustainable development have? Sustainable Development, 16(2), 81-90.

Pearce, D. W., Markandya, A., \& Barbier, E. (1989). Blueprint for a green economy (Vol. 1). Earthscan.

Perez, O., Amichai-Hamburger, Y., \& Shterental, T. (2009). The Dynamic of Corporate Self-Regulation: ISO 14001, Environmental Commitment, and Organizational Citizenship Behavior. Law \& Society Review, 43(3), 593-630.

Phillis, Y. A., \& Andriantiatsaholiniaina, L. A. (2001). Sustainability: An ill-defined concept and its assessment using fuzzy logic. Ecological Economics, 37(3), 435-456. https://doi.org/10.1016/S09218009(00)00290-1

Pinto, L. J. S., Freire, F. S., \& Dos Santos, M. A. C. (2015). Retorno das ações com status de reputação corporativa no Brasil: Um estudo comparativo entre empresas ranqueadas no MERCO e empresas listadas no ISE BM\&F BOVESPA. RAGC, 4(9).

Potoski, M., \& Prakash, A. (2013). Do voluntary programs reduce pollution? Examining ISO 14001's effectiveness across countries. Policy Studies Journal, 41(2), 273-294.

Preston, L. E., \& O'bannon, D. P. (1997). The corporate social-financial performance relationship: A typology and analysis. Business \& Society, 36(4), 419-429.

Redclift, M. (2005). Sustainable development (1987-2005): an oxymoron comes of age. Sustainable Development. Retrieved from http://onlinelibrary.wiley.com/doi/10.1002/sd.281/full

Ricardino Filho, A. ., \& Carvalho, N. (2016). Integrated Report: The Cases of Itaú Unibanco Holding S/A and of Natura Cosméticos S/A., (In: Mio C. (eds) Integrated Reporting.).

Rodrigues, S. (2010). Espiritual e sustentável. Vitória: ArtPoint.

Romeiro, A. R. (2010). Economia do Meio Ambiente: teoria e prática. Economia do Meio Ambiente e Serviços Ambientais.

Russo, M. V, \& Fouts, P. A. (1997). A resource-based perspective on corporate environmental performance and profitability. Academy of Management Journal, 40(3), 534-559. 
Sachs, J. D. (2012). From millennium development goals to sustainable development goals. The Lancet, 379(9832), 2206-2211. https://doi.org/10.1016/S0140-6736(12)60685-0

Sala, S., Farioli, F., \& Zamagni, A. (2013). Progress in sustainability science: lessons learnt from current methodologies for sustainability assessment: Part 1. The International Journal of Life Cycle Assessment, 18(9), 1653-1672.

Saunders, H. D. (2014). Toward a neoclassical theory of sustainable consumption: Eight golden age propositions. Ecological Economics, 105, 220-232. https://doi.org/10.1016/j.ecolecon.2014.06.011

Scholtens, B. (2008). A note on the interaction between corporate social responsibility and financial performance. Ecological Economics, 68(1), 46-55.

Soini, K., \& Birkeland, I. (2014). Exploring the scientific discourse on cultural sustainability. Geoforum. https://doi.org/10.1016/j.geoforum.2013.12.001

Solow, R. (1993). An almost practical step toward sustainability. Resources Policy, 19(3), 162-172. https:// doi.org/10.1016/0301-4207(93)90001-4

Stavins, R. N., Wagner, A. F., \& Wagner, G. (2003). Interpreting sustainability in economic terms: Dynamic efficiency plus intergenerational equity. Economics Letters, 79(3), 339-343. https://doi.org/10.1016/ S0165-1765(03)00036-3

Summit, E. (1992). Agenda 21. The United Nations Programme for Action from Rio.

Vallance, S., Perkins, H. C., \& Dixon, J. E. (2011). What is social sustainability? A clarification of concepts. Geoforum, 42(3), 342-348. https://doi.org/10.1016/j.geoforum.2011.01.002

Van Beurden, P., \& Gössling, T. (2008). The worth of values-a literature review on the relation between corporate social and financial performance. Journal of Business Ethics, 82(2), 407-424.

Waas, T., Hugé, J., Verbruggen, A., \& Wright, T. (2011). Sustainable Development: A Bird's Eye View. Sustainability, 3(12), 1637-1661. https://doi.org/10.3390/su3101637

Wagner, J. (2006). On the economics of sustainability. Ecological Economics, 57(4), 659-664. https://doi. org/10.1016/j.ecolecon.2005.05.023

Wartick, S. L., \& Cochran, P. L. (1985). The evolution of the corporate social performance model. Academy of Management Review, 10(4), 758-769.

Weitzman, M. L. (1997). Sustainability and technical progress. The Scandinavian Journal of Economics, 99(1), 1-13.

Wood, H. W. (1985). The United Nations World Charter for Nature: The Developing Nations' Initiative to Establish Protections for the Environment. Ecology Law Quarterly, 12(4), 977-996.

Wrigley, E. A. (2013). Energy and the English industrial revolution. Phil. Trans. R. Soc. A, 371(1986), 20110568.

Zhao, J. (2008). The effect of the ISO-14001 environmental management system on corporate financial performance. International Journal of Business Excellence, 1(1-2), 210-230. 